\title{
Dual-innervated multivector muscle transfer using two superficial subslips of the serratus anterior muscle for long-standing facial paralysis
}

\author{
Hisashi Sakuma ${ }^{1,2}$, Ichiro Tanaka ${ }^{1}$, Masaki Yazawa ${ }^{3}, \mathrm{Anna} \mathrm{Oh}^{3}$ \\ ${ }^{1}$ Department of Plastic and Reconstructive Surgery, Ichikawa General Hospital, Tokyo Dental College, Ichikawa; ${ }^{2}$ Department of Plastic and \\ Reconstructive Surgery, Yokohama Municipal Citizen's Hospital, Yokohama; ${ }^{3}$ Department of Plastic and Reconstructive Surgery, Keio \\ University School of Medicine, Tokyo, Japan
}

Recent reports have described several cases of double muscle transfers to restore natural, symmetrical smiles in patients with long-standing facial paralysis. However, these complex procedures sometimes result in cheek bulkiness owing to the double muscle transfer. We present the case of a 67-year-old woman with long-standing facial paralysis, who underwent two-stage facial reanimation using two superficial subslips of the serratus anterior muscle innervated by the masseteric and contralateral facial nerves via a sural nerve graft. Each muscle subslip was transferred to the upper lip and oral commissures, which were oriented in different directions. Furthermore, a horizontal fascia lata graft was added at the lower lip to prevent deformities such as lower lip elongation and deviation. Voluntary contraction was noted at roughly 4 months, and a spontaneous smile without biting was noted 8 months postoperatively. At 18 months after surgery, the patient demonstrated a spontaneous symmetrical smile with adequate excursion of the lower lip, upper lip, and oral commissure, without cheek bulkiness. Dual-innervated muscle transfer using two multivector superficial subslips of the serratus anterior muscle may be a good option for long-standing facial paralysis, as it can achieve a symmetrical smile that can be performed voluntarily and spontaneously.

Keywords Facial paralysis / Free tissue flap / Serratus anterior muscle
Correspondence: Hisashi Sakuma Department of Plastic and Reconstructive Surgery, Ichikawa General Hospital, Tokyo Dental College, 5-11-13, Sugano, Ichikawa 272-8513, Japan

Tel: $+81-47-322-0151$

Fax: +81-47-325-4456

E-mail: prssakuma@gmail.com

Received: August 11, 2020 • Revised: October 29, 2020 • Accepted: November 3, 2020

pISSN: 2234-6163 • elSSN: 2234-6171 • https://doi.org/10.5999/aps.2020.01599• Arch Plast Surg 2021;48:282-286

\section{INTRODUCTION}

Muscle transfer is used to reconstruct a natural, symmetrical smile in patients with long-standing facial paralysis. Although the transplanted muscle should ideally be reinnervated by the facial nerve to synchronize perioral movement with the healthy side, the healthy contralateral facial nerve alone sometimes does not obtain sufficient contraction of the transplanted muscle [1]. To solve this problem, the ipsilateral masseteric nerve was used as a neural motor source to obtain strong voluntary contraction at an early stage [2]. However, it remained difficult to reproduce an involuntary spontaneous smile synchronized with the healthy side [3-5]. In recent years, many studies reported dual innervation using a combination of the masseteric and contralateral facial nerves to compensate for this drawback. Watanabe et al. [6] first described dual innervation using the latissimus dorsi muscle innervated by the contralateral facial nerve by endto-end neurorrhaphy and the masseteric nerve via muscular 
neurotization of the underlying masseter muscle. Biglioli et al. [7] reported one-stage transfer of the gracilis muscle, innervated by both the masseteric and contralateral facial nerves. It is possible to add the ability to smile for conveying emotions through input from the contralateral facial nerve in an end-to-side fashion, while guaranteeing adequate contraction of the transplanted muscle by input from the masseteric nerve in an end-to-end fashion. However, single muscle transfer has limitations in reproducing a natural smile, since the multiple mimetic muscles have different vectors and move in coordination when smiling normally $[8,9]$. To overcome these problems, we developed a natural smile reconstruction method involving the transfer of two superficial subslips of the serratus anterior (SA) muscle, which are dually innervated by the ipsilateral masseteric and contralateral facial nerves.

\section{CASE}

A 67-year-old woman presented with complete facial paralysis that had lasted for 2 years secondary to facial nerve schwannoma resection (Fig. 1). In the first stage, through a preauricular incision on the healthy side, cross-face nerve grafting (CFNG) was performed. Two facial nerve buccal branches were identified and the thicker one was used as the recipient nerve. Six months after the first stage, dual-innervated muscle transfer was performed, using two superficial SA subslips. Through a midaxillary lazy-S incision with extension into the axilla, the ipsilateral lower SA subslips and its overlying neurovascular pedicle were identified. The 5-mm-thick and 12 -cm-long seventh and eighth superficial subslips were harvested, including the thoracodorsal vessels and long thoracic nerve, with preservation of the upper branches to prevent postoperative scapular winging (Fig. 2).

The seventh and eighth subslips were placed into the cheek
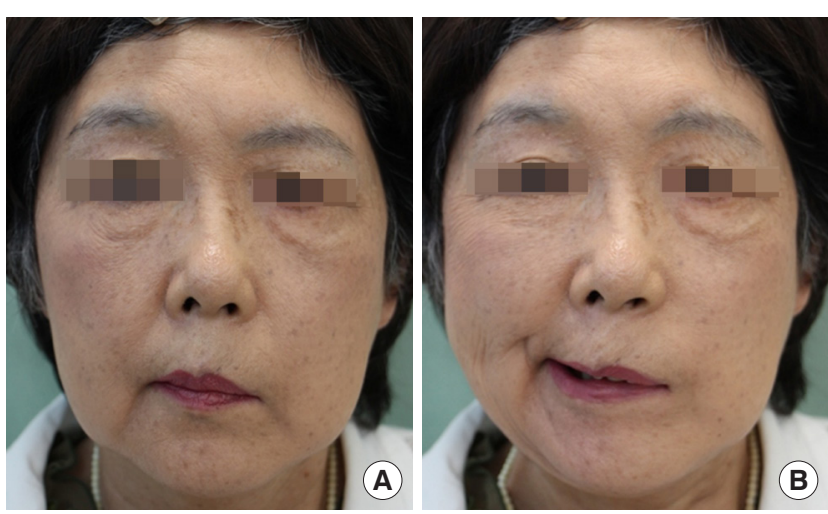

Fig. 1. A woman presenting with complete facial paralysis. (A) Preoperative view at rest. (B) Preoperative view during smiling. pocket, with the neurovascular bundles oriented on the inner side. The scapular origin of the muscle subslips was divided into two portions and positioned on the oral commissure side. After the thoracodorsal vessels were anastomosed with the facial artery and vein under a surgical microscope, the seventh subslip was secured to the orbicularis oris of the upper lip and the eighth subslip to the modiolus. The long thoracic nerve was sutured to the masseteric nerve branch in an end-to-end manner and sutured to the distal stump of the CFNG in an end-to-side manner (Fig. 3A). The opposite sides of the muscle subslip were sufficiently separated from each other to differentiate the force vector of each subslip, preserving the proper subslip vessels and nerves. The upper seventh subslip ( $8 \mathrm{~cm}$ long) was oriented to make an angle of $50^{\circ}$ and the lower eighth subslip (10 $\mathrm{cm}$ long) was positioned to make an angle of $20^{\circ}$ with the horizontal plane and secured to the deep temporal and periauricular fascia (Fig. 3B). Furthermore, through bilateral incisions along the lower vermilion border, a horizontal fascia lata graft was added between the orbicularis oris muscle of the lower lip and the lower eighth subslip to prevent deformities, such as lower lip

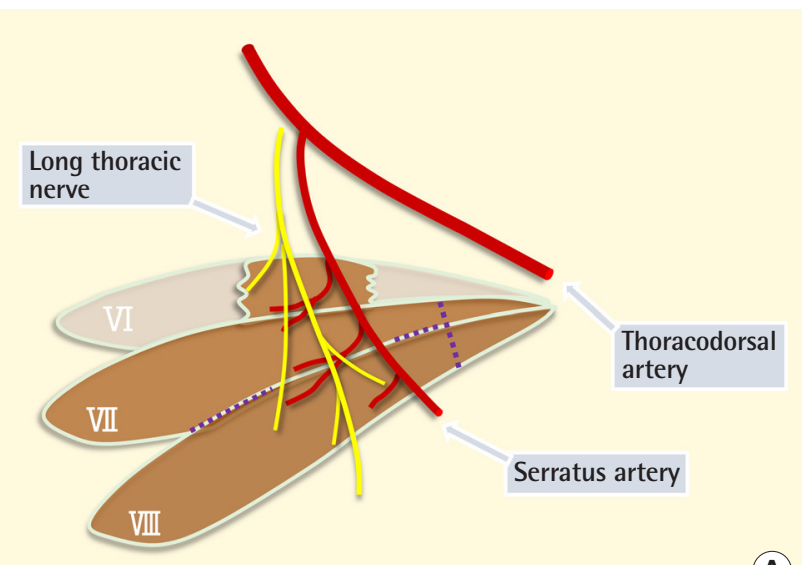

(A)

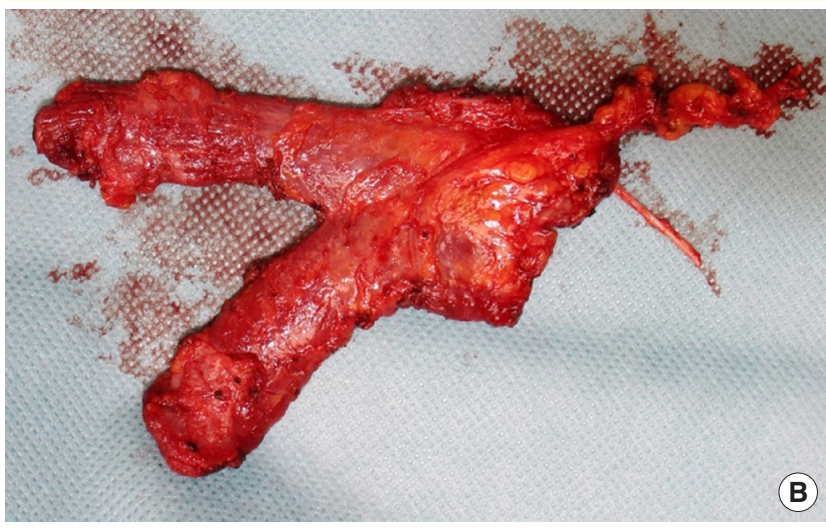

Fig. 2. Harvest of the lower serratus anterior (SA) muscle. (A) Schema of the surgical anatomy of the SA and its overlying neurovascular pedicle. (B) Two harvested superficial subslips of the SA. 


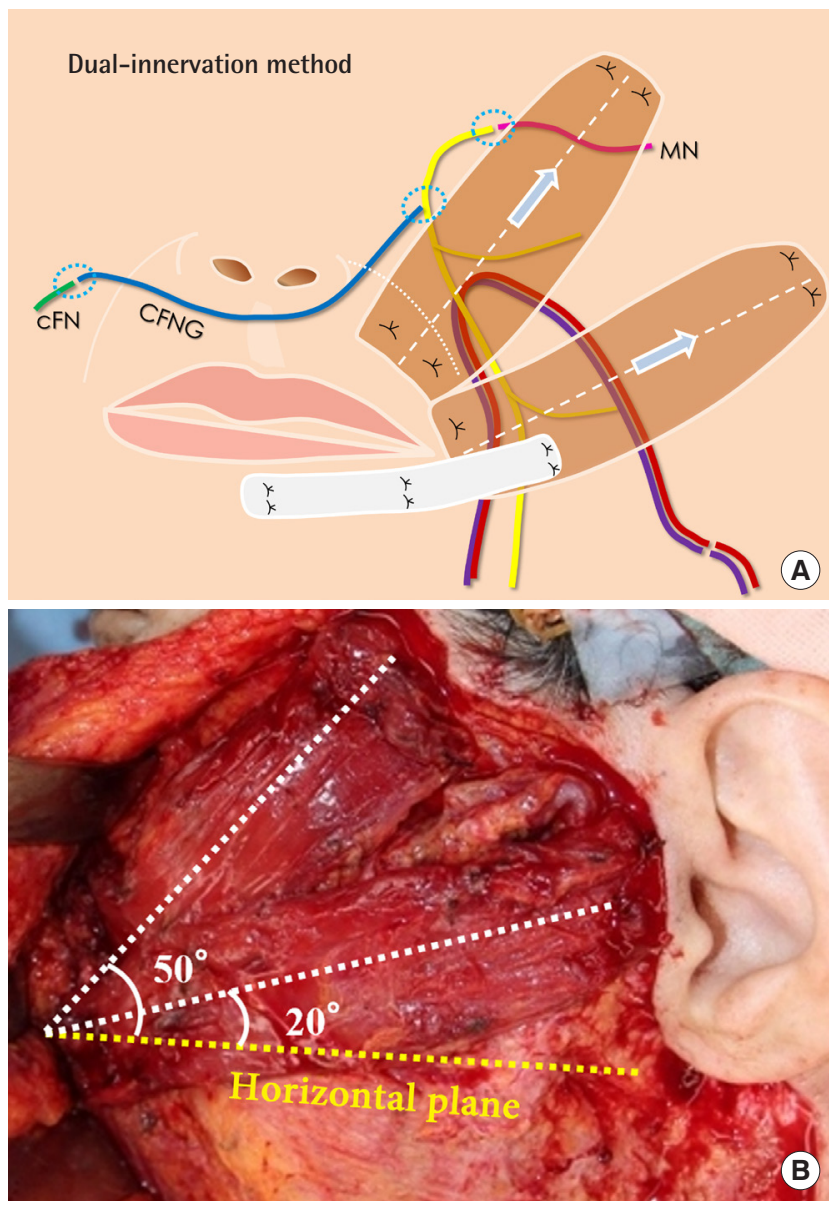

Fig. 3. Placement of the two muscle subslips. (A) Schema of the dual-innervation method. The long thoracic nerve was sutured to the branch of the MN in an end-to-end fashion and to the distal stump of the CFNG in an end-to-side fashion. The horizontal fascia lata graft was added between the lower lip and the lower eighth subslip. (B) The upper seventh subslip was oriented to make an angle of $50^{\circ}$ and the lower eighth subslip to make an angle of $20^{\circ}$ to the horizontal plane. CFN, contralateral facial nerve; $\mathrm{MN}$, masseteric nerve; CFNG, cross-face nerve graft.

thinning and elongation. No muscle necrosis, vascular complications, or donor-site problems were observed, including scapular winging.

Voluntary contraction was noted at approximately 4 months, and a spontaneous smile without biting was noted 8 months postoperatively. At 18 months after surgery, the patient simultaneously demonstrated a spontaneous symmetrical smile with sufficient excursion of the lower lip, upper lip, and oral commissure (Fig. 4). Postoperative cheek muscle bulkiness with the two subslips was not demonstrated at rest or while smiling.

\section{DISCUSSION}

In recent years, various double muscle transfer methods have
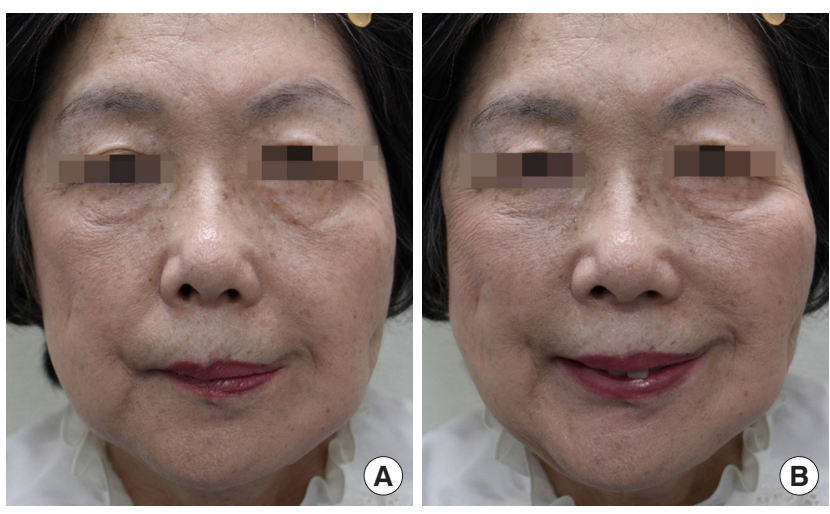

Fig. 4. Postoperative view at 18 months after surgery. (A) At rest. (B) Spontaneous smile.

been reported for long-standing facial paralysis, in which each muscle flap is reinnervated by two neural motor sources to reproduce complex perioral movements. Okazaki et al. [10] demonstrated dual latissimus dorsi muscle flap transfer with nerve suturing to the ipsilateral masseteric and contralateral facial nerves. Although this method using two muscle flaps mimics complex facial movements and guarantees reliable muscle contraction and voluntary smiling in one stage, it is difficult to produce a coordinated smile due to the independent innervation of each muscle flap. Matsumine et al. [11] reported a single-stage double muscle reconstruction technique using a latissimus dorsi muscle flap and SA flap, with dual innervation from the contralateral facial and masseteric nerves with neural interconnection via each distal nerve branch. This is an excellent technique in that coordinated natural smiles can be reconstructed by dually innervating each muscle flap. However, the two procedures mentioned above are somewhat complicated and tend to exhibit muscle bulkiness. Furthermore, it is feared that movement may be weakened by thinning of the originally thick latissimus dorsi muscle.

We focused on the SA muscle, whose muscle subslips are nourished and controlled by one neurovascular bundle. We previously reported a method of transferring two or three superficial subslips of the SA reinnervated by the masseteric nerve to the lower lip, upper lip, and oral commissure with different vectors [12]. However, due to the close proximity of the muscle subslips around the lips, a sufficient vector difference could not always be obtained, resulting in insufficient lip elevation or cheek bulkiness during muscle contraction. To address these issues, we developed a method in which only two muscle subslips were fixed to the upper lip and oral commissure, regardless of the state of paralysis and facial size, and the lower lip is pulled outward through a fascial graft. The superior muscle subslip to be transplanted is mainly aimed at raising the upper lip and 
forming the nasolabial fold, with the inferior muscle subslip abducting the oral commissure and lower lip to the outside. As a result, by synchronously pulling the two muscle subslips controlled by one nerve with sufficiently different vectors, a more natural smile can be obtained. Combining this technique with a fascial graft also makes it possible to reconstruct more esthetic smiles and prevents muscle bulkiness. Furthermore, it is possible to simply reproduce a spontaneous symmetrical smile by adding additional input from the contralateral facial nerve via a CFNG to the proximal side of the long thoracic nerve with endto-side neurorrhaphy, similar to Biglioli's procedure [7]. The disadvantages of this method are that it is a two-stage reconstruction requiring a sural nerve graft; it is expected that a spontaneous smile is unlikely to occur due to the instability of axonal regeneration from the ipsilateral facial nerve via an end-to-side anastomotic site; and excessive contraction of the transplanted muscle is likely to occur due to the inclusion of a large amount of axons from the masseteric nerve via an end-to-end anastomotic site [13].

As a result of this preliminary study, we believe that dual-innervated muscle transfer using two multivector superficial subslips of the SA muscle may be useful for the reanimation of longstanding facial paralysis because it can produce a voluntary and spontaneous smile without muscle bulkiness. Further study is necessary to confirm these findings.

\section{NOTES}

\section{Conflict of interest}

No potential conflict of interest relevant to this article was reported.

\section{Ethical approval}

The study was approved by the Institutional Review Board of the Yokohama Municipal Citizen's Hospital (IRB No. 20-11-02) and performed in accordance with the principles of the Declaration of Helsinki. Written informed consent was obtained.

\section{Patient consent}

The patient provided written informed consent for the publication and the use of her images.

\section{Author contribution}

Conceptualization: H Sakuma. Methodology: H Sakuma, I Tanaka, A Oh. Writing - original draft: H Sakuma. Writing - review \& editing: M Yazawa. Approval of final manuscript: all authors.

\section{ORCID}

Hisashi Sakuma

https://orcid.org/0000-0002-6073-6832

Ichiro Tanaka

Masaki Yazawa

Anna Oh

https://orcid.org/0000-0002-9786-1418

https://orcid.org/0000-0002-2446-2766

https://orcid.org/0000-0003-1422-2205

\section{REFERENCES}

1. Cuccia G, Shelley O, d'Alcontres FS, et al. A comparison of temporalis transfer and free latissimus dorsi transfer in lower facial reanimation following unilateral longstanding facial palsy. Ann Plast Surg 2005;54:66-70.

2. Manktelow RT, Tomat LR, Zuker RM, et al. Smile reconstruction in adults with free muscle transfer innervated by the masseter motor nerve: effectiveness and cerebral adaptation. Plast Reconstr Surg 2006;118:885-99.

3. Faria JC, Scopel GP, Busnardo FF, et al. Nerve sources for facial reanimation with muscle transplant in patients with unilateral facial palsy: clinical analysis of 3 techniques. Ann Plast Surg 2007;59:87-91.

4. Biglioli F, Colombo V, Tarabbia F, et al. Recovery of emotional smiling function in free-flap facial reanimation. J Oral Maxillofac Surg 2012;70:2413-8.

5. Banks CA, Jowett N, Iacolucci C, et al. Five-year experience with fifth-to-seventh nerve transfer for smile. Plast Reconstr Surg 2019; 143:1060e-1071e.

6. Watanabe Y, Akizuki T, Ozawa T, et al. Dual innervation method using one-stage reconstruction with free latissimus dorsi muscle transfer for re-animation of established facial paralysis: simultaneous reinnervation of the ipsilateral masseter motor nerve and the contralateral facial nerve to improve the quality of smile and emotional facial expressions. J Plast Reconstr Aesthet Surg 2009;62:1589-97.

7. Biglioli F, Colombo V, Tarabbia F, et al. Double innervation in free-flap surgery for long-standing facial paralysis. J Plast Reconstr Aesthet Surg 2012;65:1343-9.

8. Rubin LR. The anatomy of a smile: its importance in the treatment of facial paralysis. Plast Reconstr Surg 1974;53: 384-7.

9. Paletz JL, Manktelow RT, Chaban R. The shape of a normal smile: implications for facial paralysis reconstruction. Plast Reconstr Surg 1994;93:784-9.

10. Okazaki M, Kentaro T, Noriko U, et al. One-stage dual latissimus dorsi muscle flap transfer with a pair of vascular anastomoses and double nerve suturing for long-standing facial paralysis. J Plast Reconstr Aesthet Surg 2015;68:e113-9.

11. Matsumine H, Kamei W, Fujii K, et al. One-stage reconstruction by dual-innervated double muscle flap transplan- 
tation with the neural interconnection between the ipsilateral masseter and contralateral facial nerve for reanimating established facial paralysis: a report of 2 cases. Microsurgery 2019;39:457-62.

12. Sakuma H, Tanaka I, Yazawa M, et al. Multivector functioning muscle transfer using superficial subslips of the serratus anterior muscle for longstanding facial paralysis. J Plast Reconstr Aesthet Surg 2019;72:964-72.

13. Tanaka I, Sakuma H, Yazawa M, et al. Examination of the long-term course of the free muscle grafting that assumed masseteric nerve singularity motor nerves for old facial paralysis. Facial Nerve Res Jpn 2018;38:58-60. 